\title{
REDUCED GRAPHENE OXIDE AS REINFORCEMENT IN ALUMINIUM NANOCOMPOSITES PREPARED BY POWDER METALLURGY
}

\section{Daniel Andrada Maria*1, Andreza de Sousa Andrada1, Tiago de Mattos Serodre², Adelina Pinheiro Santos $^{2}$ and Clascidia Aparecida Furtado 2}

1Universidade Federal de Itajubá, Instituto de Engenharias Integradas, Campus Theodomiro Santiago - Itabira - MG, Brasil

${ }^{2}$ Centro de Desenvolvimento da Tecnologia Nuclear, Laboratório de Química de Nanoestruturas de Carbono - Belo

Horizonte - MG, Brasil

\section{ARTICLE INFO}

\section{Article History:}

Received $14^{\text {th }}$ March, 2021

Received in revised form

$03^{\text {rd }}$ April, 2021

Accepted $11^{\text {th }}$ May, 2021

Published online $20^{\text {th }}$ June, 2021

\section{Key Words:}

Nanocomposites, Aluminium,

Powder metallurgy, Nanomaterials,

Reduced graphene oxide.

*Corresponding author:

Daniel Andrada Maria

\begin{abstract}
This study investigated the use of reduced graphene oxide (rGO) obtained by chemical route as a reinforcement phase in aluminium matrix nanocomposites (AMNCs) fabricated by powder metallurgy. Mechanical milling of a mixture of aluminium and rGO powders was able to form homogeneous and highly densified composites, allowing an effective interaction between matrix and reinforcement to establish. The effect of rGO content (ranging for 0.01 to $1.0 \mathrm{wt} \%$ ) on the mechanical properties of rGO-AMNCs was investigated. Microhardness, elastic modulus and yield strength increase gradually up to $0.5 \mathrm{wt} \%$ with respective gains of $308 \%, 61 \%$ and $86 \%$.
\end{abstract}

Copyright (C) 2021, Daniel Andrada Maria et al. This is an open access article distributed under the Creative Commons Attribution License, which permits unrestricted use, distribution, and reproduction in any medium, provided the original work is properly cited.

Citation: Daniel Andrada Maria, Andreza de Sousa Andrada, Tiago de Mattos Serodre, Adelina Pinheiro Santos and Clascidia Aparecida Furtado. 2021. "Reduced graphene oxide as reinforcement in aluminium nanocomposites prepared by powder metallurgy", International Journal of Development Research, $11,(06), 47594-47598$.

\section{INTRODUCTION}

Aluminium matrix nanocomposites (AMNCs) reinforced with carbon nanomaterials have attracted great attention in the last few decades due to their potential use in different areas such as the automotive and aerospace sectors, meeting specific demands currently fulfilled exclusively by metals and their alloys (Nieto, 2017). These nanocomposite materials offer a unique balance of the physical and mechanical properties of its constituents, such as ductility, tenacity, and thermal and electrical properties of the metallic matrix, together with the high elastic modulus, and high electrical and thermal conductivity of the graphenic materials. In addition, the proportions of the phases in the metal matrix composites (MMCs) are not directly limited by thermodynamic factors as in metallic alloys, offering great possibilities in terms of properties that can be designed, customized and adapted to a given application (Bianco, 2013). Among carbon nanomaterials, carbon nanotubes (CNTs) and, more recently, all graphene family compounds (Bianco, 2013), i.e., graphene oxide (GO), reduced graphene oxide (rGO), few layer graphene (FLG), graphene nanosheets (GNS) and graphene nanoplatelets (GNPs) are considered to be the best candidates to provide AMNCs with better enhanced properties. Graphene materials seem to comprise the best choice for reinforcement in nanocomposites over other forms of carbon due to their relatively large surface area providing greater interaction with the matrix material, which implies an increased and more efficient transfer of electrons, phonons or mechanical stresses (Nieto, 2017). Many researchers have focused in attempting to incorporate graphene materials into $\mathrm{Al}$ matrix in order to obtain AMNCs with desirable properties. Despite constant efforts, the reinforcing effects are still notably lower than the theoretically predicted, due mainly to factors related with incorporation and dispersion efficiency of the graphene material in the Al matrix during processing (Baig, 2018). In the face of the different processing methodologies like powder metallurgy, colloidal processing, molecular level mixing and nanoscale dispersion, powder metallurgy by ball milling processing (BM) is widely used due to its low processing temperature which is beneficial by avoiding or controlling the harmful reaction interface, allied to the relatively low cost, reproducibility and scalability that make this processing a common choice for obtaining AMNCs. Bartolucci et al. (Bartolucci, 2011) was one of the first to report obtaining an AMNC reinforced with graphene. The authors evidenced a remarkable decrease in mechanical properties of the AMNC, which was attributed to the formation of interfacial products. In the following studies, different methodologies showed promising results regarding a gain in mechanical properties of the AMNCs, mostly reinforced with GNPs (Bartolucci, 2011; Bianco, 2013; El-Ghazaly, 2017; Jiang, 2019; Khan, 2017; Kwon, 2017; Li, 2017, 2015a; Liu, 2016; Niteesh Kumar, 2017; Pérez-bustamante, 2014; Raj, 2020; Rashad, 2014; 
Shin, 2016; Syed Nasimul Alamn, 2016; Turan, 2020; Zan Li et al., 2014). A few approaches consider AMNCs reinforced with rGO, obtained in situ by thermal reduction (trGO) during the sinterization process (Abdolhosseinzadeh, 2015; Bisht, 2017; Li, 2015b; Pérezbustamante, 2014; Sun, 2017; Zan, 2020). Due to its grapheme-like nature allied with its own characteristics, such as the high degree of exfoliation and high-throughput production process, rGO is an important candidate as reinforcement in AMNCs. Herein, rGO previously obtained by low temperature chemical route was used as a reinforcement material in AMNCs prepared by powder metallurgy. SEM and TEM analysis were conducted to evaluate microstructural changes and help ensure the desirable material modifications. Furthermore, the influence of the rGO content in the mechanical properties of the composite, such as compressive strength and microhardness, were measured and compared with those reported in related works.

\section{MATERIALS AND METHODS}

Materials: Atomized aluminium powder of globular particles with mean size of $6 \mu \mathrm{m}$ (purity $>99.5 \%$ ) was supplied by Alcoa ${ }^{\circledR}$, Brazil, and used as received. Milled expanded grafite with average lateral size of $8 \mu \mathrm{m}$ and thickness of 5-10 $\mathrm{nm}$ was supplied by Nacional de Graphite LTDA, Brazil. For simplification purposes this sample was labelled as GNP. All solvents and reagents were of analytic grade and used without further purification.

Preparation of rGO: Firstly, a graphene oxide (GO) sample was obtained from natural graphite through an adapted Hummers method described by Abdolhosseinzadeh et al.(Abdolhosseinzadeh, 2015). The sample was washed with $1 \mathrm{~mol} \cdot \mathrm{L}^{-1} \mathrm{HCl}$ solution and distilled water for several times by centrifugation (Eppendorf, 5810-R) at $10000 \mathrm{rpm}$. The final solid precipitate was dried at temperatures below $40^{\circ} \mathrm{C}$ under vacuum for 48 hours and was manually ground until it passed through a sieve of $40 \#$ to obtain a homogenous powdered sample, which was chemically reduced to rGO. This reduction was carried out in one step under reflux treatment in $\mathrm{pH} 10$ solution containing $0.45 \mathrm{~mol}^{\mathrm{L}} \mathrm{L}^{-1} \mathrm{~L}$-ascorbic acid (LAA) as reducing agent $(\mathrm{LAA}: \mathrm{GO}=10: 1)$. The solid product was separated by vacuum filtration and washed using deionized water and anhydrous ethanol. The final product was collected and dried in vacuum at temperature lower than $40^{\circ} \mathrm{C}$ for $24 \mathrm{~h}$.

Preparation of AMNCs: The AMNC powders were prepared by ball milling at $250 \mathrm{rpm}$ for $3 \mathrm{~h}$ in a $250 \mathrm{~mL}$ stainless steel jar with steel grinding balls of $2 \mathrm{~mm}$ in diameter, under inert atmosphere of ultrapure $\mathrm{N}_{2}$, using a Retch PM 100 high energy (Germany) planetary ball mill. The ball to powder weight ratio was kept constant at 10:1. Methanol $\left(\mathrm{CH}_{3} \mathrm{OH}\right)$ with purity of $99.99 \%$ was used as process control agent to avoid cold welding in a ratio of $0.8 \mathrm{wt} \%$ in relation to the aluminium content. At the end of the process, the jar with the powder blend was kept in a desiccator under vacuum to allow the passivation of the metallic powders for $24 \mathrm{~h}$. Afterwards, each powder was placed in a die of $10 \mathrm{~mm}$ in diameter and cold compacted at a compaction pressure of $550 \mathrm{MPa}$ in order to fabricate the billets. The obtained samples were placed in a tubular furnace with dry and inert gas atmosphere. The sintering conditions were: maximum temperature of $605^{\circ} \mathrm{C}$, holding time of $180 \mathrm{~min}$, and heating rate of 10 C. $\mathrm{min}^{-1}$. Samples with different weight percent of the reinforcement ranging from $0.01,0.05,0.5$ and $1.00 \mathrm{wt} \%$ were obtained. For comparison an Al sample was also prepared using the same method excluding the graphene material addition.

Characterization: Morphology and structural characterizations were performed by optical microscopy (OM; Carl Zeiss-Axio Imager A2m, Gottingen, Germany), scanning electron microscopy (SEM) (SEM, TESCAN VEGA2) and transmission electron microscopy (Tecnai G2-20 Super Twin, FEI under the acceleration voltage of 200 $\mathrm{KV})$. The relative density of the AMNC samples was measured by Archimedes' principle. The microhardness measurements were performed using a Vickers Wilson-402 MVD microdurometer with a load of $0.2 \mathrm{kgf}$ and a time of 15 seconds. Compression tests were performed at room temperature on the basis of the monolithic cylindrical samples according to ASTM E9 using an Instron 2300 universal test machine with a $100 \mathrm{KN}$ load cell with a speed of 0.5 $\mathrm{mm} \cdot \mathrm{min}^{-1}$. Cylindrical samples with an aspect ratio $(1 / \mathrm{d})$ of $\sim 1.5$ were used in all assays. At least three samples for each composition were measured to ensure the accuracy.

\section{RESULTS AND DISCUSSIONS}

Morphology of the composite powders: Before ball milling dispersion, all mixtures resulted in a fine powder with a bright surface. In this condition, the resulting powders are still very reactive and need to be handled very carefully. SEM images of the powders obtained by manual mixing show aspects of both $\mathrm{Al}$ matrix and isolated reinforcement (Fig. $1-a-b)$. Fig. 1-b clearly identifies a large flake of $\mathrm{rGO}$ attached to a non-deformed surfaces of $\mathrm{Al}$ particle before dispersion by ball milling.
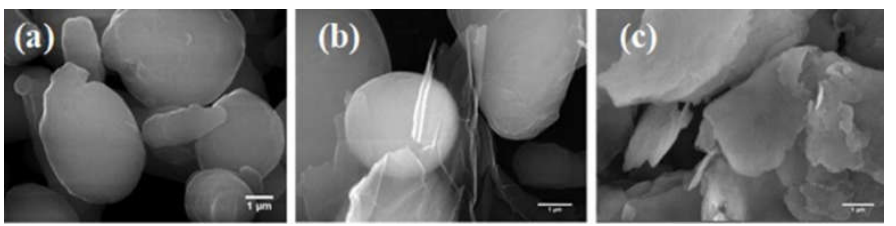

Fig. 1. SEM images of Al particle(a), $1.0 \mathrm{wt} \%$ rGO-AMNC before dispersion (b) and $1.0 \mathrm{wt} \%$ rGO-AMNC after ball milling dispersion (c)

After milling it was not possible to distinguish the phases of carbon material from the metal matrix (Figure 1-c). Particles of irregular shape resulting from cold welding, deformation and fragmentation are observed in the image. Such a change in the morphology of the particles can be considered desirable from the point of view of compaction, since in thesis they provide a better packing than spherical particles allowing to obtain a higher densification. No rGO agglomerate was observed in representative SEM images of the milling composites.

Density of AMNCs: Calculated $\left(\rho_{\mathrm{c}}\right)$ and experimental $\left(\rho_{\mathrm{e}}\right)$ density values of sintered composites were evaluated for all AMNCs and are displayed in Table 1. Calculated densities were computed by the rule of mixtures, using the experimentally measured density of each constituent of the composite material, i.e. aluminium and $\mathrm{rGO}$, found to be respectively $2.698 \pm 0.006 \mathrm{~g} . \mathrm{cm}^{-3}$ and $1.651 \pm 0.019 \mathrm{~g} . \mathrm{cm}^{-3}$. Since rGO is less dense than aluminium, a slightly reduction in the density of the composites is expected in the range of the reinforcement concentration studied here. A significant reduction should just occur with incorporating large volumes of reinforcement. For pure aluminium, a reduction of $\sim 2 \%$ in the measured density after sintering $(2.63 \pm 0.01)$ was observed in comparison to the one measured for $\mathrm{Al}$ powder. This reduction demonstrates the high level of densification that can be achieved by the proposed methodology, and is in accordance with the literature values(Khan, 2017; Rashad, 2014; Zan, 2020). For rGO-AMNCs composites, $\rho_{\mathrm{e}}$ values were $2.63 \pm 0.01,2.63 \pm 0.01,2.60 \pm 0.01$ and $2.57 \pm 0.01$ for $0.01,0.05,0.5$ and $1.0 \mathrm{wt} \% \mathrm{rGO}$ content, respectively. Above the content of 0.5 $\mathrm{wt} \%$, the incorporation of reinforcement materials has a symptomatic effect in reducing the density, reaching $\sim 96 \%$ in terms of relative $\rho_{\mathrm{e}}$. A general difference between $\rho_{\mathrm{e}}$ and $\rho_{\mathrm{c}}$ can be expected due the mixing rule neglecting any effect related to morphological, textural changes such as porosity and / or product formation (agglomeration of the reinforcement) changes, interface between aluminium and carbon, etc. Similar results were obtained by Khan et al. (Khan, 2017; Zan, 2020), who used a similar methodology to that used in this work and in other studies using other processing methodologies (Bisht, 2017; $\mathrm{Li}, 2017)$. In order to evaluate the densification of the composites by verifying of any significant porosity, the surface of rGO-AMNC was evaluated by optical microscopy (OM) and SEM. Figure 2 shows OM and SEM images for $1.0 \mathrm{wt} \% \mathrm{rGO}-\mathrm{AMNC}$, respectively, revealing an effective compaction of aluminium powders after sintering without evidences of significant porosity or rGO agglomeration. 


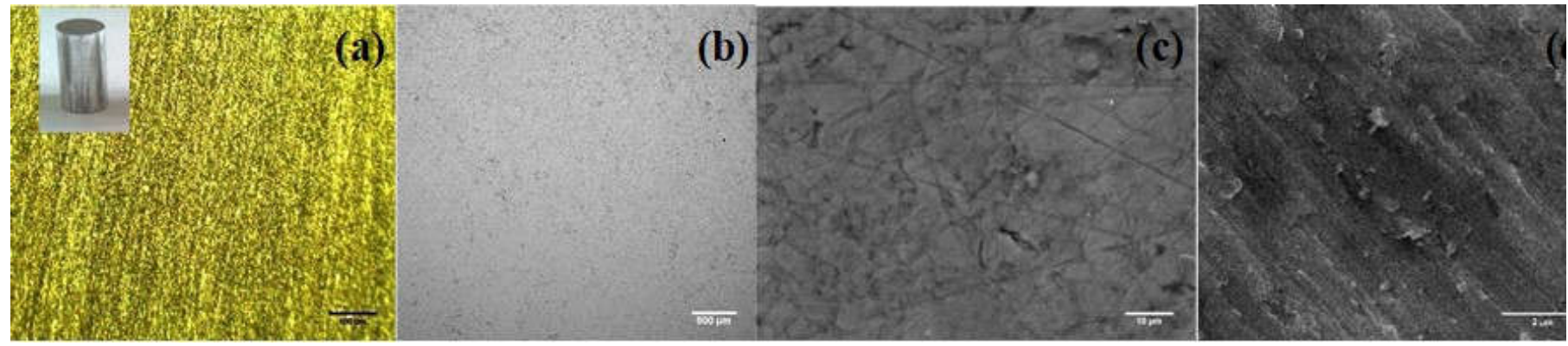

Fig. 2. Optical image of polished non-etched surfaces at 400x magnifications for $1.0 \mathrm{wt} \%$ rGO-AMNC (a) and SEM images under different magnifications (b-d) for $1.0 \mathrm{wt} \%$ rGO- AMNC
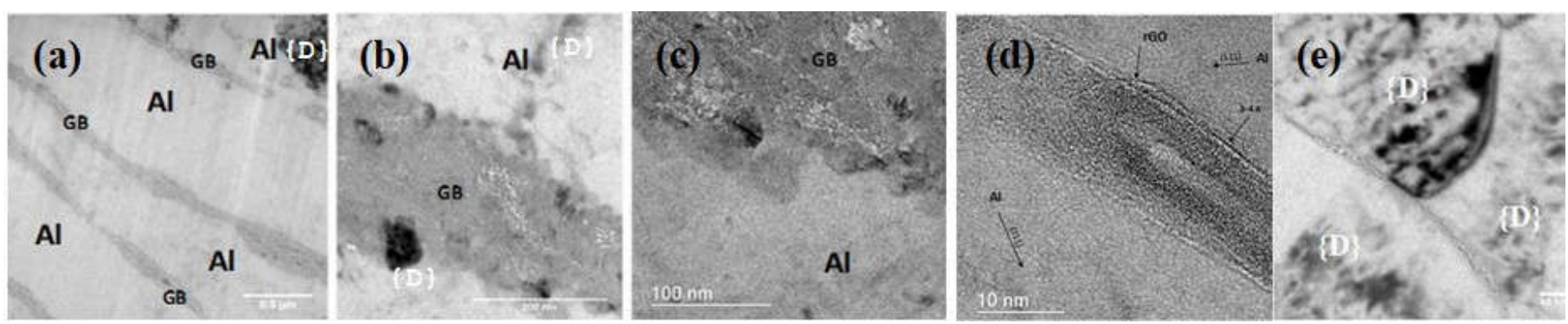

Fig. 3. TEM images under different magnifications for the $1 \mathrm{wt} \%$ rGO-AMNC sample

Table 1. Mean values of $\rho_{\mathrm{c}}, \rho_{\mathrm{e}}, \mathrm{HV}, 0.2 \%$ YS, E and UCS for the rGO-AMNC

\begin{tabular}{|l|l|l|l|l|l|l|}
\hline $\mathrm{wt} \%$ & $\rho_{\mathrm{c}}\left(\mathrm{g} \cdot \mathrm{cm}^{-3}\right)$ & $\rho_{\mathrm{e}}\left(\mathrm{g} \cdot \mathrm{cm}^{-3}\right)$ & $\mathrm{HV}$ & $0.2 \% \mathrm{YS}(\mathrm{MPa})$ & $\mathrm{E}(\mathrm{GPa})$ & UCS $(\mathrm{MPa})$ \\
\hline 0 & $2.63 \pm 0.01$ & 2.698 & $39 \pm 2$ & $57 \pm 2$ & $4.4 \pm 0,2$ & $299 \pm 16$ \\
\hline 0.01 & $2.63 \pm 0.02$ & 2.698 & $43 \pm 3$ & $57 \pm 3$ & $5.1 \pm 0,5$ & $305 \pm 5$ \\
\hline 0.05 & $2.63 \pm 0.01$ & 2.697 & $47 \pm 3$ & $71 \pm 1$ & $5.1 \pm 0,4$ & $255 \pm 3$ \\
\hline 0.5 & $2.60 \pm 0.01$ & 2.689 & $120 \pm 4$ & $106 \pm 4$ & $7.1 \pm 0,2$ & $284 \pm 2$ \\
\hline 1.0 & $2.57 \pm 0.01$ & 2.681 & $98 \pm 2$ & $99 \pm 5$ & $6.6 \pm 1,0$ & $274 \pm 11$ \\
\hline
\end{tabular}

Table 2. Comparison between the HV values reported here and in the literature for graphene content AMNCs

\begin{tabular}{|c|c|c|c|}
\hline Reinforcement & Mass fraction & Microhardness variation & Reference \\
\hline $\operatorname{trGO}$ & $0-0.1 \%$ wt. & $\sim 1.2 \mathrm{x}$ increase in $\mathrm{HV}(0.1 \% \mathrm{wt})$. & (Bartolucci, 2011) \\
\hline GNPs & $0-3 \%$ wt. & $\sim 1.7 \mathrm{x}$ increase in $\mathrm{HV}(3.0 \%$ wt. $)$ & (Latief, 2011) \\
\hline GNPs & $0-5 \%$ wt. & $\sim 1.6 \mathrm{x}$ increase in $\mathrm{HV}(5.0 \%$ wt. $)$ & (El-Ghazaly, 2017) \\
\hline GNPs & $0-5 \%$ wt. & $\sim 3.0 \mathrm{x}$ increase in $\mathrm{HV}(1.0 \% \mathrm{wt})$. & (Syed Nasimul Alamn, 2016) \\
\hline GO & $1.0 \%$ vol. & $\sim 2.4 \mathrm{x}$ increase in $\mathrm{HV}(1.0 \%$ vol. $)$ & (Kwon, 2017) \\
\hline trGO & $0-5 \%$ wt. & $\sim 1.2 \mathrm{x}$ increase in $\mathrm{HV}(0.5 \%$ wt. $)$ & (Sun, 2017) \\
\hline GNPs e trGO & $0-5 \%$ wt. & $\sim 1.4 \mathrm{x}$ increase in $\mathrm{HV}(0.3 \%$ wt. $)$ & (Liu, 2016) \\
\hline GNPs & $0-5 \%$ wt. & $\sim 1.6 \mathrm{x}$ increase in $\mathrm{HV}(1.5 \%$ wt. $)$ & (Baig, 2018) \\
\hline GNPs & $0-5 \%$ wt. & $\sim 1.3 \mathrm{x}$ increase in $\mathrm{HV}(0.5 \% \mathrm{wt})$. & (Turan, 2020) \\
\hline GO & $0-0.2 \%$ wt. & $\sim 1.1 \mathrm{x}$ increase in $\mathrm{HV}(0.2 \%$ wt. $)$ & (Dasari, 2018) \\
\hline GNPs & $0-1.0 \%$ wt. & $\sim 1.9 \mathrm{x}$ increase in $\mathrm{HV}(1.0 \%$ wt. $)$ & (Raj, 2020) \\
\hline $\mathrm{rGO}$ & $0.5 \%$ wt. & $\sim 3.0 \mathrm{x}$ increase in $\mathrm{HV}(0.5 \% \mathrm{wt})$ & Present work \\
\hline
\end{tabular}

Microhardness and compression tests of the AMNCs: Table 1 displays the values of Vickers microhardness (HV) and the parameters of compression modulus (E), $0.2 \%$ yield strength (YS) and ultimate compressive strength (UCS) obtained from the stressstrain curves for the rGO-AMNC composites in different mass fractions. In general, it was observed that HV, YS and E gradually increases by increasing the reinforcement content until the limit of $0.5 \mathrm{wt} \%$ of $\mathrm{rGO}$ and then decrease at $1.0 \mathrm{wt} \%$. The $0.2 \%$ YS parameter is a measure of the resistance of the material to flow or to being submitted to plastic deformation. An increase of up to $86 \%$ in YS at $0.5 \% \mathrm{wt} \%$. rGO agrees with the expectative of increase in mechanical resistance of the composite material. Furthermore, the increase of $61 \%$ of the elastic modulus reflects the synergetic combination of the elastic modulus of the matrix and the reinforcement, which can be reached when the material responds as a composite. Thus, we can consider that although we did not observe a clear trend or real gain in the UCS, in agreement with the results of Rashad et al.(Rashad, 2014), YS and E parameters provided evidences that rGO can act as an effective reinforcement in AMNCs. Similar behaviour was obtained in other studies reporting an enhancement in the mechanical properties of $\mathrm{Al}$ nanocomposites via favourable dispersion at low mass fraction up to $0.5 \mathrm{wt} \%$ of graphene material (Baig, 2018; Li, 2015a; Sun, 2017). However, the gain of $\sim 3$ times $(308 \%)$ in $\mathrm{HV}$ observed here for $0.5 \mathrm{wt} \% \mathrm{rGO}-\mathrm{AMNC}$, from $39 \mathrm{HV}$ (382.5 MPA) for pure Al to $120 \mathrm{HV}$ (1177 MPA), had not been reported until now. Table 2 compares our result with the others reported in the literature. Few works have reached near the maximum gain observed in this work, despite using a relatively higher amount of reinforcement (El-Ghazaly, 2017; Kwon, 2017; Syed Nasimul Alamn, 2016).

Microstructure of the AMNCs: In order to evaluate the interface and the phases present in the obtained composites, TEM analysis was carried out from thin sections obtained for the rGO-AMNCs sample. Figure 3 shows a sequence of images obtained for the $1 \mathrm{wt} \% \mathrm{rGO}-$ AMNC sample in different magnifications. Initially it is possible to identify elongated $\mathrm{Al}$ grains (Figure 3 (a)), which are presented in this way because of the milling of the powders, which cause a deformation of the Al particles, changing their original rounded shape into elongated plates with a thickness of $\sim 600 \mathrm{~nm}$ and with a higher 
width of $6 \mu \mathrm{m}$ (not shown). Regions of grain boundaries (GB) as well as the occurrence of some dislocations in the $\mathrm{Al}$ matrix $(\{\mathrm{D}\})$ can be observed in Figure 3 (a). Pores or voids were not identified in the TEM images, again characterizing a well densified sample. GB are present as discontinuous phases in the Al matrix. Similar results were observed by Zhang et al.(Jiang, 2019; Li, 2015b; Zan, 2020). Due to its intrinsic characteristics as relative low crystallinity, high exfoliation degree, thin morphology, allied to its relative low mass fraction in the AMNCs, rGO phase becomes an object of difficult observation, mainly if it is intercalated between GB or surfaces of adjacent $\mathrm{Al}$ particles. However, in some specific regions of the sample outside the grain boundary region, it was possible to identify the $\mathrm{rGO}$ phase in the form of thin curved or folded layers (Figure 3 (c) e (d)), showing that the rGO phase was introduced into the $\mathrm{Al}$ matrix and does not occupy only the border regions between the metal particles. The reinforcement phase incorporated into the matrix reflects the conditions where stress transfer from the matrix to the reinforcement can be activated. Moreover, Figure 3 (d) illustrates the tendency of occurrence of discordances $\{\mathrm{D}\}$ pilling up in the interface formed between the Al matrix and the rGO layer. The rGO act as an effective barrier to the movement of dislocations, thus controlling and restricting the plastic deformation of the matrix. Also, the $\mathrm{rGO}$ was found dispersed in the $\mathrm{Al}$ matrix and in suitable conditions to allow stress transfer phenomena to occur. These observations agree with the results obtained of increase in microhardness and their overall macroscopic mechanical properties. Due their own characteristics, such as the high degree of exfoliation, high dispersibility and relative higher interfacial strength(Chen, 2021; Li, 2018; Zhang, 2021) than other near-pristine or high-quality graphene materials like GNPs, the rGOcan act as effective reinforcement phase in AMNCs.

\section{CONCLUSIONS}

Chemical reduced GO (rGO) was employed as reinforcement in AMNCs. Composites with uniformly dispersed rGO in loadings of 0.01 to $1.0 \mathrm{wt} \%$ were successfully manufactured by powder metallurgy technique using the ball milling processes. Uniaxial pressing and pressureless sintering were used for the densification of mixed powders into compact composite bodies. The degree of densification of the sintered composites was of $\sim 96 \%$. Pores and voids were not identified by TEM, which also characterized the rGO phase acting as a barrier to the movement of dislocations and fully inserted into the metallic matrix in an appropriate condition for the phenomena of stress transfer. Additionally, intermetallic compounds like $\mathrm{Al}_{4} \mathrm{C}_{3}$ were not evidenced, signaling a good interaction between the reinforcement and the metal matrix. As a consequence, gains in mechanical properties of $61 \%$ in elastic modulus, $86 \%$ in yield strength and $308 \%$ in microhardness were reached for rGO-AMMCs at the limit of $0.5 \mathrm{wt} \% \mathrm{rGO}$ content.

\section{ACKNOWLEDGMENT}

This work was supported by the Brazilian Nanocarbon Institute, the SisNano Program, and the Brazilian agencies CNPq, CAPES, FAPEMIG, and CNEN. The authors would like to acknowledge Alcoa and Nacional de Grafite LTDA for the supply of samples, Dr. D.R. Miquita and Dr. H. Limborço from Center of Microscopy of UFMG for insightful work in TEM/FIB analysis.

\section{REFERENCES}

Abdolhosseinzadeh, S., Asgharzadeh, H., \& Kim, H. S. 2015. Fast and fully-scalable synthesis of reduced graphene oxide. Scientific Reports, 5: 1-7.

Baig, Z., Mamat, O., Mustapha, M., Mumtaz, A., Sarfraz, M., \& Haider, S. 2018. An Efficient Approach to Address Issues of Graphene Nanoplatelets (GNPs) Incorporation in Aluminium Powders and Their Compaction Behaviour. Metals, 8(2): 90.
Bartolucci, S. F., Paras, J., Rafiee, M. A., Rafiee, J., Lee, S., Kapoor, D., \& Koratkar, N. 2011. Graphene-aluminum nanocomposites. Materials Science and Engineering A, 528(27): 7933-7937.

Bianco, A., Cheng, H. M., Enoki, T., Gogotsi, Y., Hurt, R. H., Koratkar, N., Kyotani, T., Monthioux, M., Park, C. R., Tascon, J. M. D., \& Zhang, J. 2013. All in the graphene family - A recommended nomenclature for two-dimensional carbon materials. Carbon, 65: 1-6.

Bisht, A., Srivastava, M., Manoj, R., Lahiri, I., \& Lahiri, D. 2017. Strengthening mechanism in graphene nanoplatelets reinforced aluminum composite fabricated through spark plasma sintering. Materials Science \& Engineering A.

Chen, Y., Liu, Y., Zhou, F., Chen, M., Qu, N., Liao, M., \& Zhu, J. 2021. The interface properties of defective graphene on aluminium: A first-principles calculation. Computational Materials Science, 188(December 2020): 110157.

Dasari, B. L., Morshed, M., Nouri, J. M., Brabazon, D., \& Naher, S. 2018. Mechanical properties of graphene oxide reinforced aluminium matrix composites. Composites Part B: Engineering, 145(October 2017): 136-144.

El-Ghazaly, A., Anis, G., \& Salem, H. G. 2017. Effect of graphene addition on the mechanical and tribological behavior of nanostructured AA2124 self-lubricating metal matrix composite. Composites Part A: Applied Science and Manufacturing, 95: 325-336.

Jiang, Y., Xu, R., Tan, Z., Ji, G., Fan, G., Li, Z., Xiong, D. B., Guo, Q., Li, Z., \& Zhang, D. 2019. Interface-induced strain hardening of graphene nanosheet/aluminum composites. Carbon, 146: 1727.

Khan, M., Amjad, M., Khan, A., Ud-Din, R., Ahmad, I., \& Subhani, T. 2017. Microstructural evolution, mechanical profile, and fracture morphology of aluminum matrix composites containing graphene nanoplatelets. Journal of Materials Research, 32(11): 2055-2066.

Kwon, H., Mondal, J., AloGab, K., Sammelselg, V., Takamichi, M., Kawaski, A., \& Leparoux, M. 2017. Graphene oxide-reinforced aluminum alloy matrix composite materials fabricated by powder metallurgy. Journal of Alloys and Compounds, 698: 807-813.

Latief, F. H., Sherif, E. M., Almajid, A. A., \& Junaedi, H. 2011. Journal of Analytical and Applied Pyrolysis Fabrication of exfoliated graphite nanoplatelets-reinforced aluminum composites and evaluating their mechanical properties and corrosion behavior. Journal of Analytical and Applied Pyrolysis, 92(2): 485-492.

Li, G., \& Xiong, B. 2017. Effects of graphene content on microstructures and tensile property of graphene-nanosheets / aluminum composites. Journal of Alloys and Compounds, 697: $31-36$.

Li, J. L., Xiong, Y. C., Wang, X. D., Yan, S. J., Yang, C., He, W. W., Chen, J. Z., Wang, S. Q., Zhang, X. Y., \& Dai, S. L. 2015 a. Microstructure and tensile properties of bulk nanostructured aluminum/graphene composites prepared via cryomilling. Materials Science and Engineering A, 626: 400-405.

Li, Z., Fu, X., Guo, Q., Zhao, L., Fan, G., Li, Z., Xiong, D. B., Su, Y., \& Zhang, D. 2018. Graphene quality dominated interface deformation behavior of graphene-metal composite: The defective is better. International Journal of Plasticity, 111: 253265.

Li, Z. Z., Guo, Q., Li, Z. Z., Fan, G., Xiong, D., Su, Y., Zhang, J., Zhang, D., Zan Li, Qiang Guo*, Zhiqiang Li, Genlian Fan, Ding-Bang Xiong, Y. S., \& Jie Zhang, D. Z. 2015b. Enhanced Mechanical Properties of Graphene (Reduced Graphene Oxide)/Aluminum Composites with a Bioinspired Nanolaminated Structure. Nano Letters, 15: 1-19.

Liu, J., Khan, U., Coleman, J., Fernandez, B., Rodriguez, P., Naher, S., \& Brabazon, D. 2016. Graphene oxide and graphene nanosheet reinforced aluminium matrix composites: Powder synthesis and prepared composite characteristics. Materials and Design, 94: 87-94. 
Nieto, A., Bisht, A., Lahiri, D., Zhang, C., \& Agarwal, A. 2017. Graphene reinforced metal and ceramic matrix composites: a review. International Materials Reviews, 62(5): 241-302.

Niteesh Kumar, S. J., Keshavamurthy, R., Haseebuddin, M. R., \& Koppad, P. G. 2017. Mechanical Properties of AluminiumGraphene Composite Synthesized by Powder Metallurgy and Hot Extrusion. Transactions of the Indian Institute of Metals.

Pérez-bustamante, R., Bolaños-morales, D., Bonilla-martínez, J., \& Estrada-guel, I. 2014. Microstructural and hardness behavior of graphene-nanoplatelets / aluminum composites synthesized by mechanical alloying. Journal of Alloys and Compounds, 615: S578-S582.

Raj, R. R., Yoganandh, J., Saravanan, M. S. S., \& Kumar, S. S. 2020. Effect of graphene addition on the mechanical characteristics of AA7075 aluminium nanocomposites. Carbon Letters, (0123456789).

Rashad, M., Pan, F., Tang, A., \& Asif, M. 2014. Effect of Graphene Nanoplatelets addition on mechanical properties of pure aluminum using a semi-powder method. Progress in Natural Science: Materials International, 24(2): 101-108

Shin, S. E., Ko, Y. J., \& Bae, D. H. 2016. Mechanical and thermal properties of nanocarbon-reinforced aluminum matrix composites at elevated temperatures. Composites Part B: Engineering.
Sun, Y., Zhang, C., Liu, B., Meng, Q., Ma, S., \& Dai, W. 2017. Reduced Graphene Oxide Reinforced 7075 Al Matrix Composites: Powder Synthesis and Mechanical Properties. Metals, 7(11): 499.

Syed Nasimul Alamn, L. K. 2016. A Mechanical properties of aluminium based metal matrix composites reinforced with graphite nanoplatelets. Materials Science \& Engineering A, 667: $16-32$.

Turan, M. E., \& Aydin, F. 2020. Improved elevated temperature mechanical properties of graphene-reinforced pure aluminium matrix composites. Materials Science and Technology (United Kingdom), 36(10): 1092-1103.

Zan $\mathrm{Li}$ et al. 2014. Uniform dispersion of graphene oxide in aluminum powder by direct electrostatic adsorption for fabrication of graphene / aluminum composites. Nanotechnology, 25(32): 325601.

Zan, Y. N., Zhang, Q., Zhou, Y. T., Liu, Z. Y., Wang, Q. Z., Wang, D., Xiao, B. L., Ren, W. C., \& Ma, Z. Y. 2020. Introducing graphene (reduced graphene oxide) into $\mathrm{Al}$ matrix composites for enhanced high-temperature strength. Composites Part B: Engineering, 195(April): 1-10.

Zhang, X., \& Wang, S. 2021. Interfacial strengthening of graphene/aluminum composites through point defects: A firstprinciples study. Nanomaterials, 11(3): 1-16. 